\title{
High-Resolution NMR of Anisotropic Samples With Spinning Away from the Magic Angle
}

\author{
Dimitris Sakellariou, Carlos A. Meriles, Rachel W. Martin and Alexander Pines*
}

March 31, 2003

Department of Chemistry, UC Berkeley, Berkeley, CA 94720, USA

to be submitted to Chem. Phys. Lett.

\begin{abstract}
High-resolution NMR of samples in the solid state is typically performed under mechanical sample spinning around an axis that makes an angle, called the magic angle, of 54.7 degrees with the static magnetic field. There are many cases in which geometrical and engineering constraints prevent spinning at this specific angle. Implementations of in-situ and ex-situ magic angle field spinning might be extremely demanding because of the power requirements or an inconvenient sample size or geometry. Here we present a methodology based on switched angle spinning between two angles, none of which is the magic angle, which provides both isotropic and anisotropic information. Using this method, named Projected Magic Angle Spinning, we were able to obtain resolved scaled isotropic chemical shifts in spinning samples where the broadening is mostly inhomogeneous.
\end{abstract}

\section{Introduction}

The introduction of Magic Angle Spinning (MAS) in NMR [1,2], allowed high-resolution spectra of samples subject to anisotropic line broadening. Under fast spinning of the

\footnotetext{
${ }^{*}$ To whom correspondence should be addressed.
} 
sample around an axis that makes a specific angle of $\theta_{m}=\arctan \sqrt{2} \approx 54.7^{\circ}$ with the static magnetic field $B_{0}$, named the Magic Angle, a significant line narrowing occurs. The time dependent spatial part of the anisotropic interactions averages over a rotor period and the time independent component is canceled by the choice of the angle. Currently, MAS is applied routinely in most high-resolution solid-state NMR experiments, and spinning frequencies continue to increase dramatically with increasing $B_{0}[3]$.

The idea, first proposed by Andrew [4], of spinning the field at the magic angle represents an alternative for static samples located inside (in-situ) or outside (ex-situ) of the magnet and has recently been the focus of considerable attention $[5,6]$. Here the constraint of reaching the magic angle represents serious engineering problems, since in the case of spinning the field electromagnetically or rotating a permanent magnet, the required power increases substantially with the angle [7]. In addition, there are cases where even mechanically spinning the sample exactly at the magic angle represents a problem due to possible geometrical constraints, e.g. elongated sample that cannot be spun inside the bore of a superconducting magnet, or even induced disorder e.g. liquid crystals [8].

Here we present a method based on Switched Angle Spinning (SAS) to obtain highresolution two-dimensional spectra containing isotropic-anisotropic correlations. Data were collected on samples rotating at angles different from the magic angle, restricted between $0^{\circ}$ and $54.7^{\circ}$ (MAS). SAS has been used in the past to obtain similar correlations, with the constraint of one angle being the magic angle [9-12], and also in the context of Dynamic Angle Spinning (DAS) [13,14] for high resolution NMR of quadrupolar nuclei. Our goals here are quite different; we are interested in recording the full isotropic and anisotropic spectroscopic information by spinning the sample or the magnetic field around axes making angles with the static magnetic field away from the magic angle. Since the Larmor 
frequencies are much higher than the spinning frequencies, the adiabatic limit is satisfied and spinning of the field is essentially equivalent to spinning the sample [4]. The power requirements of spinning a field become less stringent when the angle of the rotation is lower. From Fig. 1 one can estimate that stronger magnetic fields generated by electromagnets can be rotated faster at the expense of a smaller angle of rotation. Keeping in mind the existing techniques, our goal here is to develop the methodology for the equivalent off-magic angle spinning field experiments.

In this paper, the first implementation of projected-MAS (p-MAS) is shown. Spectra of different samples subject to susceptibility broadening are recorded while spinning the sample away from the magic angle. The two-dimensional spectra provide correlations between the anisotropic and the scaled isotropic chemical shifts. Extensions of the method to onedimensional p-MAS and projected-Magic Angle Turning versions are discussed together with the limitations of the approach.

\section{Projected-Magic Angle Spinning}

In what follows we treat samples characterized by inhomogeneous [15] anisotropic broadening, including such mechanisms as magnetic susceptibility, heteronuclear dipolar coupling and chemical shift anisotropy. All of these interactions are assumed to transform like rank two spatial tensors upon sample rotation. ${ }^{1}$ The spin Hamiltonian for such spin systems can be written as:

$$
H(t ; \theta)=\sum_{m=-2}^{2} \exp \left(-i m \omega_{r} t\right) H_{m}(\theta)
$$

\footnotetext{
${ }^{1}$ The line-broadening coming from homogeneous or higher rank components in the Hamiltonian will be discussed later in this paper.
} 
Making the assumption that the spinning frequency is much higher than the anisotropic broadening, only the term that corresponds to $m=0$ is relevant. This term is responsible for the width of the centerband and can be expressed as:

$$
H_{0}(\theta)=H_{\text {iso }}+\mathcal{P}_{2}(\cos \theta) H_{\text {aniso }}
$$

where $\mathcal{P}_{2}(\cos \theta)$ is the rank two Legendre polynomial. The two dimensional p-MAS experiment consists of a free evolution period during time $t_{1}$ with the sample spinning at an angle $\theta_{1}$, followed by the acquisition during time $t_{2}$ at an angle $\theta_{2}$. The pulse sequence is depicted schematically in Fig. 2(a). The hopping of the rotor from $\theta_{1}$ to $\theta_{2}$ and its stabilization takes some finite time of the order of several ms. Thus, between the two evolution periods a storage period $\tau$ is inserted in order to preserve the transverse signal as longitudinal magnetization, which decays more slowly. Proper phase cycling [14] selects the coherence pathways shown in Fig. 2(b) allowing frequency sign discrimination in both dimensions. This pulse scheme leads to a two-dimensional spectrum of correlations between two anisotropic dimensions, with width determined by the values of the respective Legendre polynomials for $\theta_{1}$ and $\theta_{2}$. Nevertheless, resolution is recovered with an appropriate projection or "shearing" which produces isotropic chemical shifts scaled by a scaling factor $\lambda$. The slope of the projection depends on the ratio of the polynomials for the two angles. The narrow ridges in the two-dimensional frequency spectrum are equivalent to echoes of the anisotropic part of the Hamiltonian in the time domain. This can be easily seen from the one-dimensional equivalent of the sequence presented in Fig. 2(c). There the $\pi$ pulses refocus the evolution of the magnetization giving rise to echoes of the chemical shift anisotropy for the proper choice of $\tau_{1}$ and $\tau_{2}$. In this pulse sequence the switching between the angles $\theta_{1}$ and $\theta_{2}$ is assumed to be infinitely rapid, a limit that might be easier to achieve for field spinning compared to sample spinning. The $2 \mathrm{D}$ data can also be 
sheared and represented in the form of ordinary isotropic-anisotropic correlations. In order to observe a refocusing of the anisotropy, the anisotropic evolution (dephasing) must be the same in both dimensions:

$$
\tau_{1} \mathcal{P}_{2}\left(\cos \theta_{1}\right)=\tau_{2} \mathcal{P}_{2}\left(\cos \theta_{2}\right)
$$

On the other hand, the net evolution after time $\tau_{1}+\tau_{2}$ of the isotropic chemical shift is:

$$
\sigma_{\text {iso }}\left(\tau_{1}-\tau_{2}\right)=\sigma_{\text {iso }}\left(\tau_{1}+\tau_{2}\right) \frac{\tau_{1}-\tau_{2}}{\tau_{1}+\tau_{2}}=\sigma_{\text {iso }}\left(\tau_{1}+\tau_{2}\right) \frac{1-\tau_{1} / \tau_{2}}{1+\tau_{1} / \tau_{2}}
$$

and using Eq 3, one can calculate the scaling factor from the refocusing conditions to be:

$$
\lambda=\frac{\mathcal{P}_{2}\left(\cos \theta_{1}\right)-\mathcal{P}_{2}\left(\cos \theta_{2}\right)}{\mathcal{P}_{2}\left(\cos \theta_{1}\right)+\mathcal{P}_{2}\left(\cos \theta_{2}\right)}
$$

A representation of the scaling factor for different pairs of spinning angles is shown in Fig. 3. The resolution is dependent not only on the difference between the switching angles, but also on the absolute value of these angles, rendering the scaling extremely small for angles close to zero degrees. Of course, to the extent that the residual linewidth also scales with the angle this is not a problem. We mention here that related two-dimensional correlations experiments of amorphous solids exhibiting inhomogeneous broadening, can yield highly resolved projections (see [16] and references therein).

\section{Experiments}

All data were acquired on a Chemagnetics Infinity Spectrometer operating at $500 \mathrm{MHz}{ }^{1} \mathrm{H}$ frequency, using an extensively modified Varian $4 \mathrm{~mm}$ SAS probe. The probe design is such that the solenoidal radio-frequency coil stays parallel to the rotor at any angle, rendering the excitation and detection at an angle close to 0 degrees not efficient. The filling factor 
and the rf homogeneity are comparable to any commercial MAS probe. The samples were spun at approximately $7 \mathrm{kHz}$ and the pulse sequence of Fig. 2(a) was implemented using a $\tau$ period of 60 to $80 \mathrm{~ms}$ for the stator switching and stabilization depending on the difference of the switching angles [17]. The precision of the angle setting was estimated to be around 0.1 degrees. The magic angle was calibrated by maximizing the number of spinning sidebands on the spectrum of polycrystalline $\mathrm{KBr}$.

Two samples exhibiting proton anisotropic susceptibility broadening were analyzed. A sample, used in the literature [18] as a model for susceptibility broadening in living tissues was prepared by using glass beads with sizes between 210 and $250 \mu \mathrm{m}$. The beads were soaked with water and mineral oil and then packed inside the rotor. The spectrum of the static sample has a linewidth of $7 \mathrm{kHz}$, while the residual linewidth under MAS was $80 \mathrm{~Hz}$. The second sample was a Berea sandstone rock sample also containing water and mineral oil. The residual linewidth of this sample under MAS was $500 \mathrm{~Hz}$. The origin of this linewidth, though not fully understood, could be due to other broadening mechanisms such as $T_{2}$ relaxation and/or other components of the magnetic susceptibility. In particular, the distribution of pore sizes could lead to a distribution of isotropic susceptibility that cannot be averaged away [19]. The contribution of the rank four component of the susceptibility is averaged out by MAS as shown in Ref. [3].

In Fig. 4 we present an experimental 2D p-MAS spectrum of the beads sample, together with the projections along the two anisotropic dimensions. The angles were set to $\theta_{1}=4.5^{\circ}$ and $\theta_{2}=26^{\circ}$, providing a theoretical scaling factor of 0.16 for the isotropic chemical shift. After a shearing transformation one can obtain a trace displaying the isotropic spectrum as shown. The two resonances from water (left) and mineral oil (right) can be clearly distinguished. 
The experimental data after 2D Fourier transform are presented in Fig.5 for the sample of Berea sandstone rock saturated with water and mineral oil. MAS spectra have been recorded in the literature and show a dramatic increase in resolution over those of the static sample [20]. In Fig. 5 the angle $\theta_{1}$ was set to $33.0^{\circ}$ and $\theta_{2}$ to $47.5^{\circ}$. We also recovered the isotropic information while using $\theta_{1}=4.5^{\circ}$ and $\theta_{2}=47.5^{\circ}$ (data not shown). These experimentally observed scaling values compare well with the ones obtained from Eq. 5 of 0.67 and 0.47 respectively. The residual linewidth of this sample is much greater than that of the beads sample, making the experiment more sensitive to the scaling of the isotropic chemical shift.

\section{Discussion}

One condition of the previous treatment is that the spinning frequency was considered greater than the magnitude of the anisotropic interaction. As a result the time dependent parts of the Hamiltonian were not included and spinning sidebands are absent. In the case of field spinning, estimates of the achievable spinning frequencies depend on the size of the rotating components and thus on the angle of the conical trajectory of the total field. However, we do not expect frequencies much higher than several $\mathrm{kHz}$ to be easily achievable, rendering the problem of spectral resolution non-trivial because of the presence of spinning sidebands. Rotor synchronized techniques, such as Magic Angle Hopping [21] or Turning [22-24] have been developed in the past and could be easily combined into the framework of experiments away from the magic angle. This type of techniques eliminate the presence of sidebands even in the case of very slow spinning [25]. This does not represent a serious problem for the technique and we plan to show that with the appropriate hardware such combined techniques can recover the isotropic information even in the presence of the 
very slow spinning. Work in building a synchronized switched angle turning probe is in progress.

Another experimental issue we encountered involved the design of the probe. The use of a solenoidal coil which is coaxial with the rotor excludes efficient excitation and detection at angles close to zero. Other designs that allow excitation and detection of a spinning sample at zero degrees have been developed in the past e.g. [17], and we are currently working on improving our apparatus to include such designs. However, for the future application of spinning the magnetic field off the magic angle and close to zero, this does not present a problem. In this case one would expect an increase in signal to noise since rotating close to zero would allow us to use higher total magnetic fields. On the other hand, the magnitude of the scaling factor compared to the residual homogeneous linewidth is expected to be the limiting factor of this approach. A future extension of this technique to one-dimensional schemes, as shown in Fig. 2(c), is envisaged in our laboratory. Since it would require stroboscopic acquisition during fast reorientation of the spinning axis, such 1D versions would be particularly well adapted for field spinning experiments. Along the same ideas, Variable Angle Spinning [26] could also offer an interesting alternative. Its limited angle version is currently under development.

We have presented a proof of principle that spinning away from the magic angle, and even close to zero degrees with respect to $B_{0}$, can in some cases dramatically improve the resolution and produce isotropic-anisotropic correlation spectra. Projected Magic Angle Spinning can be applied in cases where spinning the sample or the field at the magic angle is inaccessible. Further developments along the previously mentioned lines are in progress and will be described in future work. 


\section{Acknowledgments}

This work was supported by the Director, Office of Science, Office of Basic Energy Sciences, Materials Sciences Division, of the U.S. Department of Energy under Contract No. DEAC03-76SF00098. 


\section{References}

[1] E. R. Andrew, A. Bradbury and R. G. Eades, Nature 182 (1958) 1659.

[2] I. J. Lowe, Phys. Rev. Lett. 2 (1959) 285.

[3] A. Samoson, T. Tuherm and Z. Gan, Solid State Nuclear Magnetic Resonance 20 (2001) 130 .

[4] E. R. Andrew and R. G. Eades, Discuss. Faraday Soc. 34 (1962) 38.

[5] C. A. Meriles, D. Sakellariou, H. Heise, A. J. Moulé and A. Pines, Science 293 (2001) 82.

[6] C. A. Meriles, D. Sakellariou and A. Pines, Chem. Phys. Lett. 358 (2002) 391.

[7] R. C. Gupta, Indian Journal of Pure \& Applied Physics 13 (1975) 39.

[8] J. Courtieu, D. W. Alderman, D. M. Grant and J. P. Bayles, J. Chem. Phys. 77 (1982) 723.

[9] A. Bax, N. M. Szeverenyi and G. E. Maciel, J. Magn. Reson. 55 (1983) 494.

[10] T. Terao, T. Fujii, T. Onodera and A. Saika, Chem. Phys. Lett. 107 (1984) 145.

[11] T. Terao, H. Miura and A. Saika, J. Chem. Phys. 85 (1986) 3816.

[12] A. C. Kolbert, P. J. Grandinetti, M. Baldwin, S. B. Prusiner and A. Pines, J. Phys. Chem. 98 (1994) 7936.

[13] A. Llor and J. Virlet, Chem. Phys. Lett. 152 (1988) 248. 
[14] K. T. Mueller, B. Q. Sun, G. C. Chingas, J. W. Zwanziger, T. Terao and A. Pines, J. Magn. Reson. 86 (1990) 470.

[15] M. M. Maricq and J. S. Waugh, J. Chem. Phys. 70 (1979) 3300.

[16] D. Sakellariou, S. P. Brown, A. Lesage, S. Hediger, M. Bardet, A. Meriles, A. Pines and L. Emsley, J. Am. Chem. Soc. (2003) in press, DOI: 10.1021/ja0292389.

[17] K. T. Mueller, G. C. Chingas and A. Pines, Rev. Sci. Instrum. 62 (1991) 1445.

[18] J. Z. Hu and R. A. Wind, J. Magn. Reson. 159 (2002) 92.

[19] M. Alla and E. Lippmaa, Chem. Phys. Lett. 87 (1982) 30.

[20] T. M. de Swiet, M. Tomaselli, M. D. Hürlimann and A. Pines, J. Magn. Reson. 133 (1998) 385 .

[21] A. Bax, N. M. Szeverenyi and G. E. Maciel, J. Magn. Reson. 52 (1983) 147.

[22] J. Z. Hu, D. W. Alderman, C. Ye, R. J. Pugmire and D. M. Grant, J. Magn. Reson. A 105 (1993) 82.

[23] J. Z. Hu, W. Wang, F. Liu, M. S. Solum, D. W. Alderman, R. J. Pugmire and D. M. Grant, J. Magn. Reson. A 113 (1995) 210.

[24] E. A. Hill and J. P. Yesinowski, J. Chem. Phys. 106 (1997) 8650.

[25] J. Z. Hu, D. N. Rommereim and R. A. Wind, Magn. Reson. Med. 47 (2002) 829.

[26] L. Frydman, G. C. Chingas, Y. K. Lee, P. J. Grandinetti, M. A. Eastman, G. A. Barrall and A. Pines, J. Chem. Phys. 97 (1992) 4800. 
Fig. 1: Diagram representing the power requirements necessary to obtain an electromagnetically spinning magnetic field at a given angle. Three perpendicular pairs of coils are assumed, one of which is static and the other two drive oscillating currents. The normalization is made assuming that $100 \%$ is the power needed for the magic angle. From the diagram one can appreciate the dramatic drop in power requirements when the angle is close to zero.

Fig. 2: (a) Pulse sequence for Switched Angle Spinning Experiments. The phase cycling [14] is such that only the coherence pathway diagram presented in (b) contributes to observable signal. (c) One-dimensional equivalent sequence. Switching of the spinning angles is assumed infinitely fast and stroboscopic point-by-point acquisition takes place at the echos of the chemical shift anisotropy.

Fig. 3: Scaling factor for the isotropic chemical shift interaction as a function of the two switching angles. Notice how quickly the scaling factor drops in the region close to zero degrees, making p-MAS particularly challenging.

Fig. 4: Two-Dimensional p-MAS spectrum of a sample containing glass beads saturated with water and mineral oil. The angles $\theta_{1}$ and $\theta_{2}$ were set to 4.5 and 25.0 degrees respectively. $128 t_{1}$ increments with 32 averaged scans each were recorded. The switching delay $\tau$ was set to $50 \mathrm{~ms}$. The experimental scaling factor was 0.15 . The total experimental time was $18 \mathrm{~min}$.

Fig. 5: Two-Dimensional p-MAS spectrum of a Berea rock sample containing water and mineral oil. The angles $\theta_{1}$ and $\theta_{2}$ were set to $33.0^{\circ}$ and $47.5^{\circ}$ and the switching delay was equal to $70 \mathrm{~ms}$. The two isotropic resonances can be easily identified. The experimental scaling factor was 0.4 . 
High-Resolution NMR of Anisotropic Samples With Spinning Away from the Magic Angle Sakellariou et. al.

Fig. 1

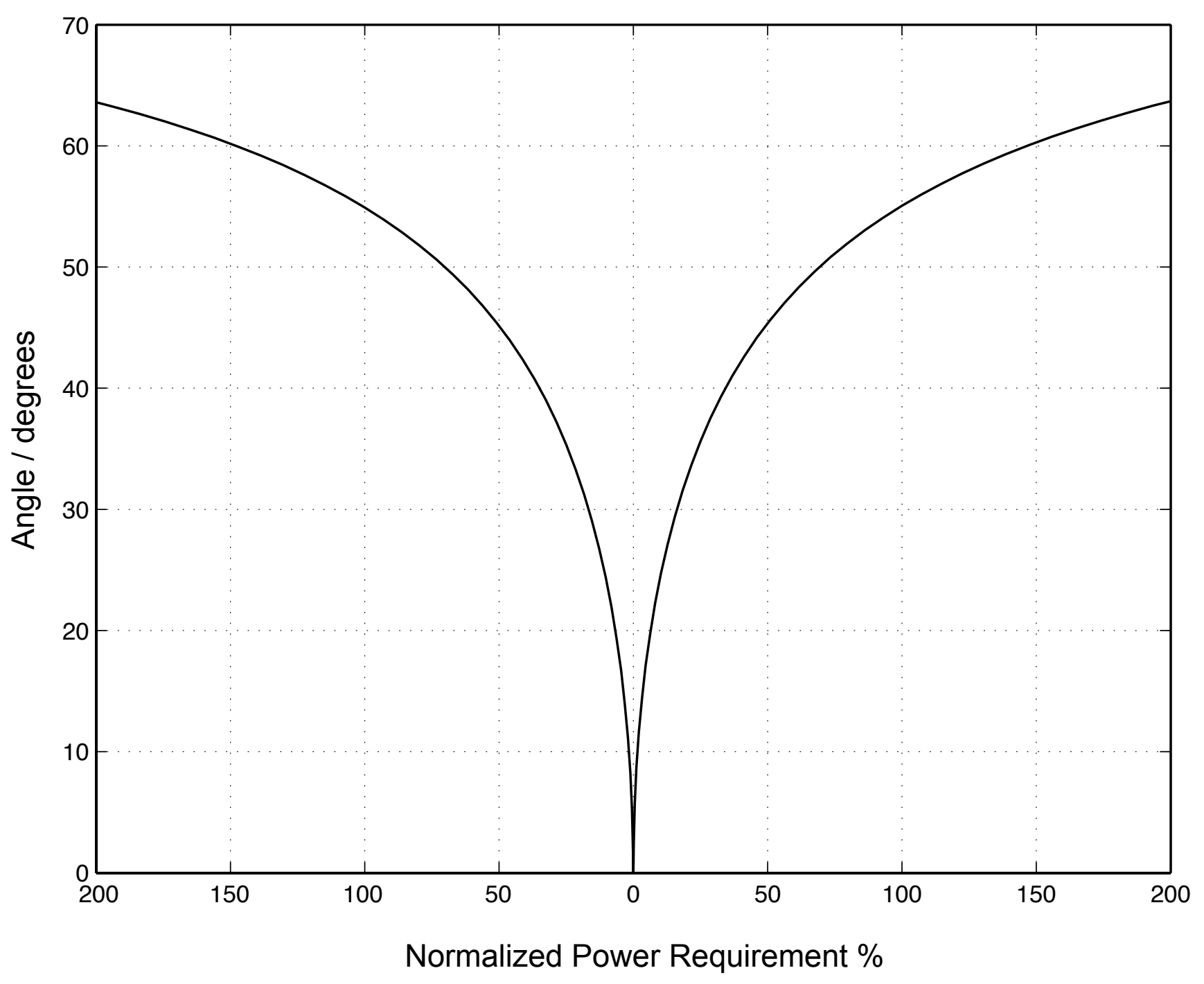


High-Resolution NMR of Anisotropic Samples With Spinning Away from the Magic Angle Sakellariou et. al.

Fig. 2

(a)
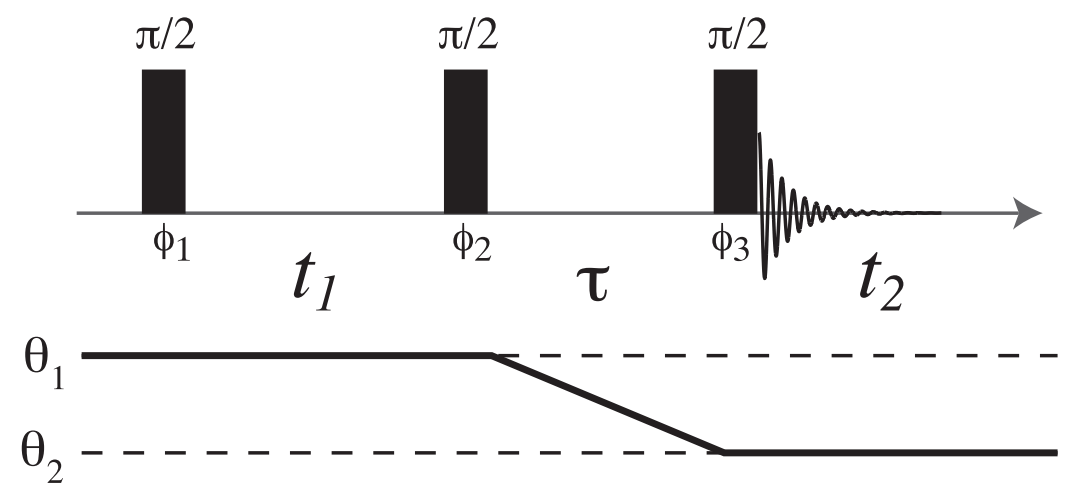

(b)

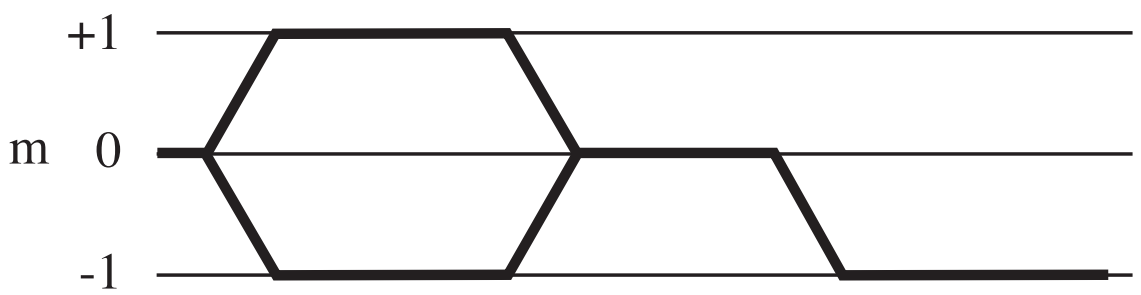

(c)
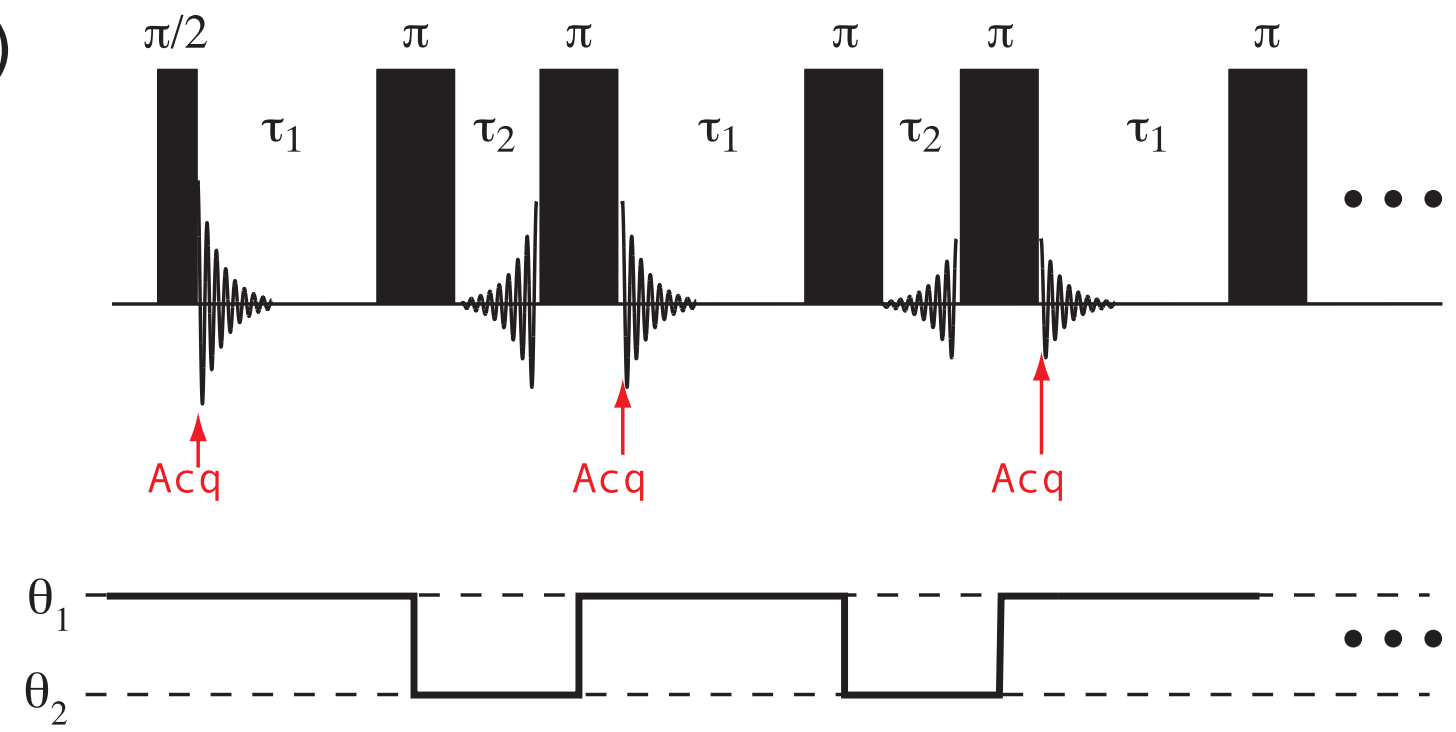
High-Resolution NMR of Anisotropic Samples With Spinning Away from the Magic Angle Sakellariou et. al.

Fig. 3

Scaling Factor

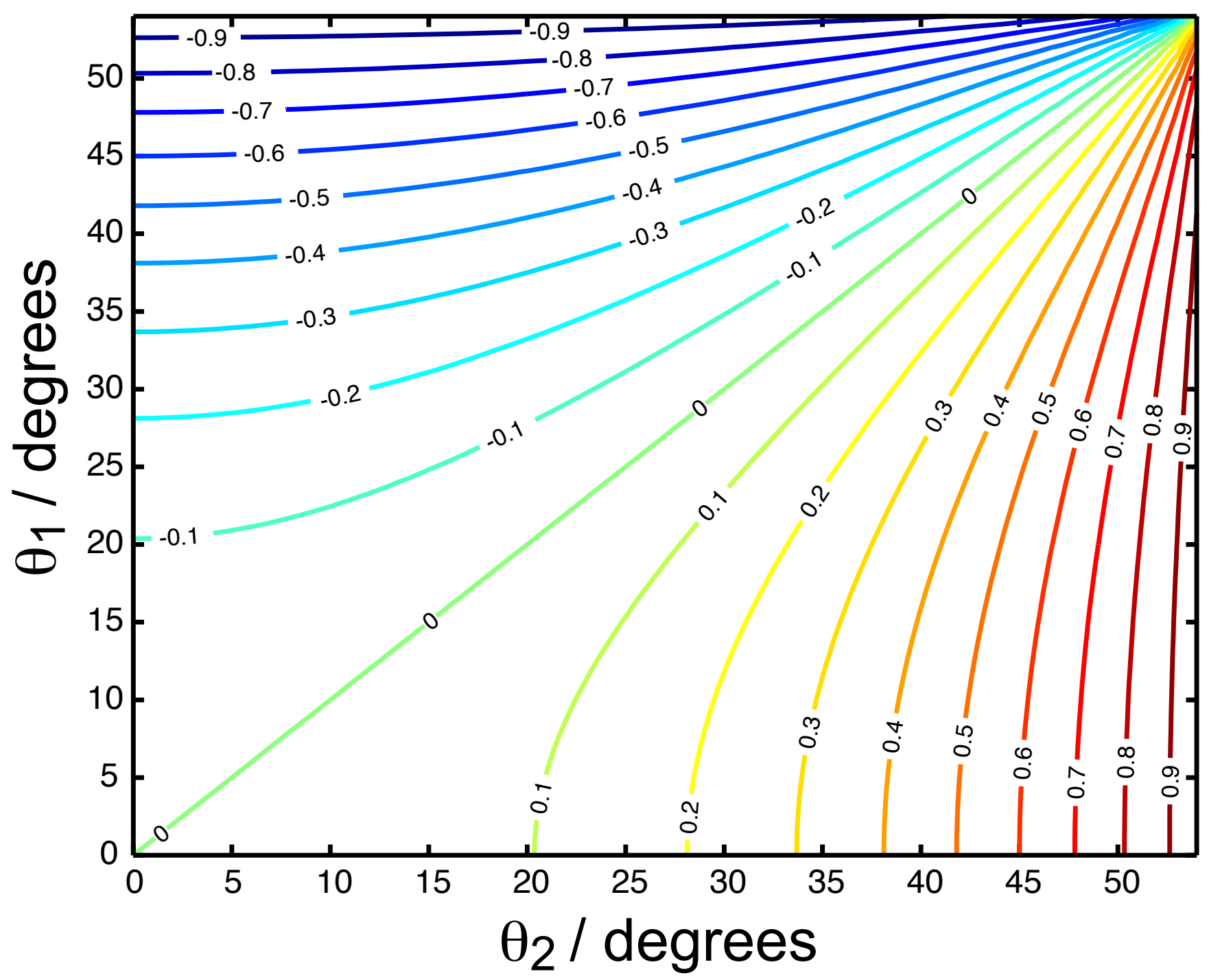


High-Resolution NMR of Anisotropic Samples With Spinning Away from the Magic Angle Sakellariou et. al.

Fig. 4

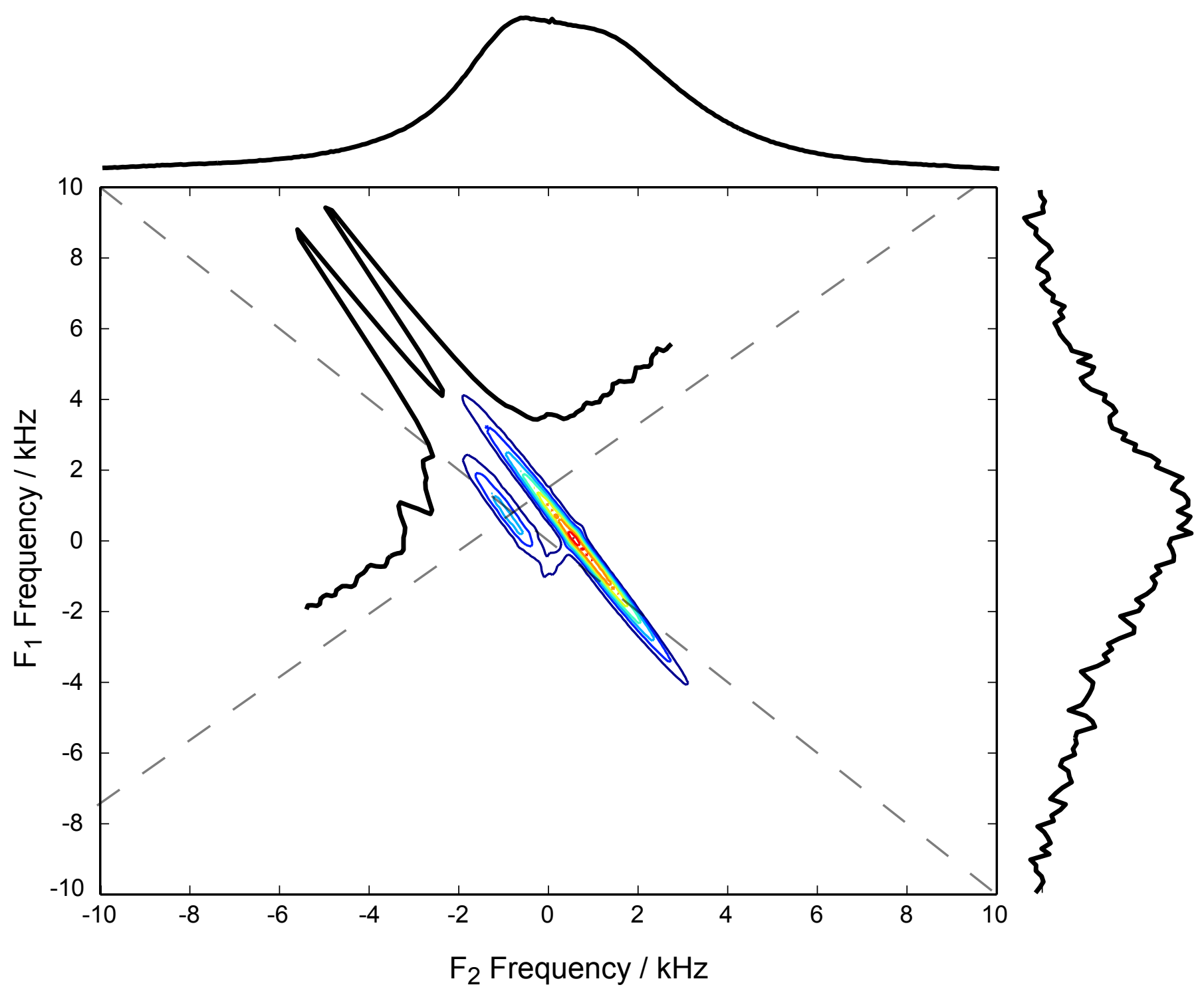


High-Resolution NMR of Anisotropic Samples With Spinning Away from the Magic Angle Sakellariou et. al.

Fig. 5

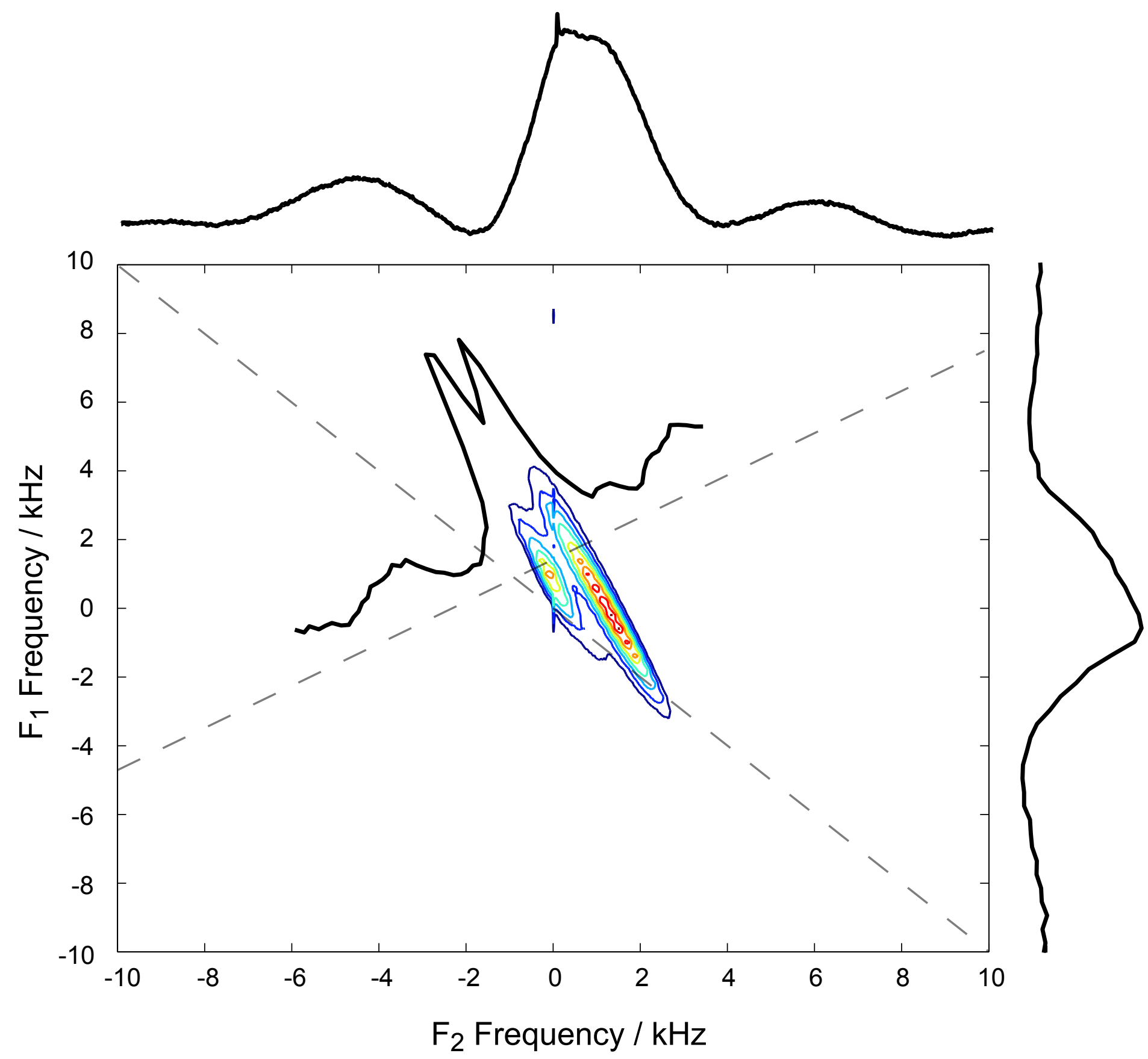

